\section{Christoph Bachmann}

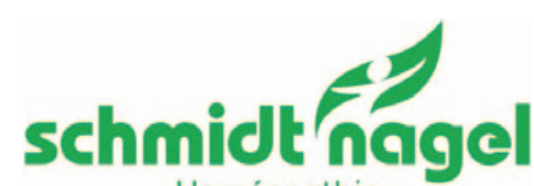

Homéopathie

\section{$+$}

Abb. 1. Das Logo von Schmidt-Nagel SA.

\section{Die Tradition von Kent in Genf}

Heute wissen nur noch wenige Leute, dass in der homöopathischen Firma Laboratoire D. Schmidt-Nagel

\title{
Das Laboratoire D. Schmidt-Nagel: Homöopathie-Tradition in Genf
}

In Meyrin, einem Vorort von Genf, befindet sich die Homöopathie-Firma Laboratoire D. Schmidt-Nagel (Abb. 1) und führt seit 1927 die Tradition der Klassischen Homöopathie weiter. Seit Kurzem ist ein studierter Ökonom Geschäftsführer, der Schmidt-Nagel, wie die Firma meistens genannt wird, in eine wirtschaftlich sichere Zukunft führen möchte.

in Genf die Tradition des grossen homöopathischen Arztes James Tyler Kent (1849-1916) weiterlebt. Der Schweizer Arzt Dr. Pierre Schmidt reiste 1927 in die USA und studierte dort Homöopathie bei Ärzten, die ihr Wissen von Kent erworben hatten. Nach seiner Rückkehr liess Schmidt sich 1930 in Genf nieder, wo seine
Tab. 1. Geschichte des Laboratoire D. Schmidt-Nagel
Heirat des Schweizer homöopathischen Arztes Dr. Pierre Schmidt mit der Apothekerin Dora Nagel.

Das Ehepaar beginnt in Genf, homöopathische Hochpotenzen herzustellen: Dies war die Geburtsstunde der späteren Firma Laboratoire D. Schmidt-Nagel SA. Anfänglich arbeitete das Ehepaar in denselben Räumen, in denen sich später auch die Arztpraxis von Pierre Schmidt befand. Damit war Schmidt-Nagel also der einzige Hersteller von Homöopathika in der Schweiz, der nicht in einer Apotheke herstellte, sondern eine eigenständige Firma war. Für die Herstellung von Hochpotenzen verwendete Dora Schmidt-Nagel einen vom Ingenieur Perdrisat entwickelten Dynamisator.

Das Laboratoire D. Schmidt-Nagel SA wird gegründet.

Dora Schmidt-Nagel gibt altersbedingt die Leitung der Firma an zwei junge Apothekerinnen; eine von ihnen, Dominique Massotte, ist die heutige Eigentümerin. Das Unternehmen wird im Geiste von Pierre und Dora Schmidt-Nagel weitergeführt.

Die Firma zieht an ihren heutigen Standort, in ein Industriegebäude an die Rue Pré-Bouvier 27 in Meyrin, einem Vorort von Genf. Dort ist mehr Platz für die Fabrikation, und die Räumlichkeiten entsprechen den heutigen Anforderungen, die an die Herstellung von Homöopathika gestellt wurden.

Die Gesellschafterin von Dominique Massotte verlässt den Betrieb, der bis 2014 von dieser alleine weitergeführt wird. mit Dominique Massotte den Betrieb.
Frau Dora Schmidt-Nagel eine homöopathische Firma, das heutige Laboratoire D. Schmidt-Nagel, gegründet hatte (Tab. 1).

\section{Schmidt-Nagel heute}

\section{Klassische Homöopathie}

Mehr als 90 Jahre nach den ersten Anfängen präsentiert sich die Schmidt-Nagel als ein moderner Hersteller von hauptsächlich homöopathischen Einzelpotenzen, die als Granuli (gross) oder Globuli (klein), Tropfen, Trinkampullen, Zäpfchen, Cremen und Salben oder Pulver angeboten werden (Abb. 2, 3).

Die Granuli und Globuli werden auf der Basis von Saccharose hergestellt und sind somit laktosefrei. Bei Unverträglichkeit auf Saccharose können diese auf Xylit-Basis bestellt werden.

\section{Weitere Präparate}

$\mathrm{Da}$ es aber schwierig ist, ein Unternehmen nur mit dem Verkauf von homöopathischen Einzelpotenzen auf Erfolgskurs zu halten (siehe Interview mit Geschäftsführer Damien Verrier (Abb. 4) in Kasten 1), hat Frau Dominique Massotte, die Eigentümerin von Schmidt-Nagel, schon vor einigen Jahren begonnen, das Geschäftsfeld auszuweiten. Heute bietet die Firma auch

\section{KARGER}

Fax +497614520714 (c) 2015 S. Karger GmbH, Freiburg 


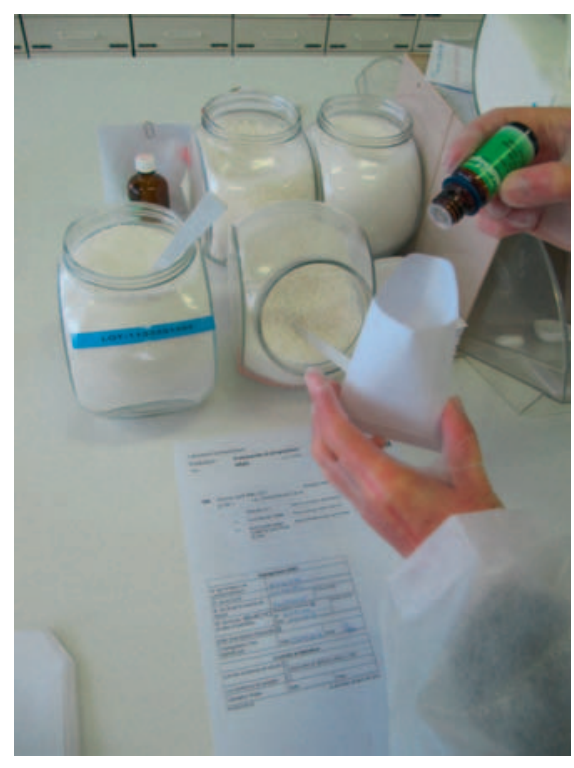

Abb. 2. Arbeit im Laboratoire Schmidt-Nagel.

alle Oligoelemente und einige Nahrungsergänzungsmittel an. Da wären z.B. ein Präparat mit rein natürlichem Vitamin C, ein Präparat auf der Basis von Wachteleierextrakt und Zink-Bisglycinat zur Stärkung des Immunsystems sowie ein Getränkepulver mit Goji, das ebenfalls das Immunsystem stärkt und die Müdigkeit bekämpft.

Zur äusserlichen Anwendung stellt Schmidt-Nagel auch drei verschiedene Cremen her. Die eine enthält Arnica-Urtinktur, die bei Blutergüssen und anderen Verletzungen nützlich ist. Die zweite wird auf der Basis von Wachteleiern hergestellt, wirkt entzündungshemmend bei Allergien und schützt die Haut gegen schädliche Umwelteinflüsse. Die dritte, eine CalendulaCreme, behandelt trockene, gereizte Haut sowie Risse und Schrunden.

\section{Einzelpotenzen}

Die Herstellung und der Vertrieb von homöopathischen Einzelpotenzen sind und bleiben aber das Kerngeschäft von Schmidt-Nagel - aus historischen Gründen und aus Überzeugung von Dominique Massotte. So werden etwa 1700 homöopathische Substanzen in verschiedensten Potenzen angeboten.

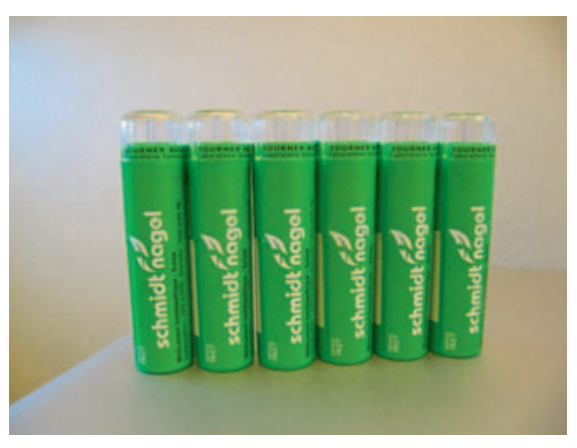

Abb. 3. Die bekannten Schmidt-Nagel-Röhrchen.

Fast alle Substanzen im Sortiment werden auch in sehr hohen Potenzen angeboten, die bis zu MM gehen können, was in der homöopathischen Nomenklatur 1 Million heisst. Ab der tausendsten Potenz werden Homöopathika mit römischen Ziffern bezeichnet.

Ebenso findet man im Sortiment LM- oder Q-Potenzen, eine spezielle Art von Potenzen, mit denen Samuel Hahnemann, der Begründer der Homöopathie, in späteren Jahren immer mehr behandelt hat.

Die Hahnemann-Potenzen, nach der Mehrglasmethode hergestellt, gibt es als D-Potenzen bis zur 60. Potenz und als C-Potenzen bis zur 1000. Potenz.

Die Korsakoff-Potenzen, nach der Einglasmethode hergestellt, gibt es von der 30. bis zur 1000 000. Potenz.

Q-Potenzen gibt es je nach Mittel bis zur 60. Potenz.

Ungefähr 300 der Substanzen sind auch als Urtinkturen erhältlich.

\section{Gemmotherapie}

Ein eher junges Betätigungsfeld von Schmidt-Nagel ist die Gemmotherapie. In den letzten Jahren ist die Nachfrage nach dieser Art von Präparaten gestiegen. Dabei handelt es sich um eine spezielle Art von pflanzlichen Zubereitungen, die mit den Knospen der entsprechenden Pflanzen hergestellt werden. Gemmo ist aus dem Lateinischen und heisst Knospe. So findet man im Sortiment von SchmidtNagel etwa 50 gemmotherapeutische Präparate.

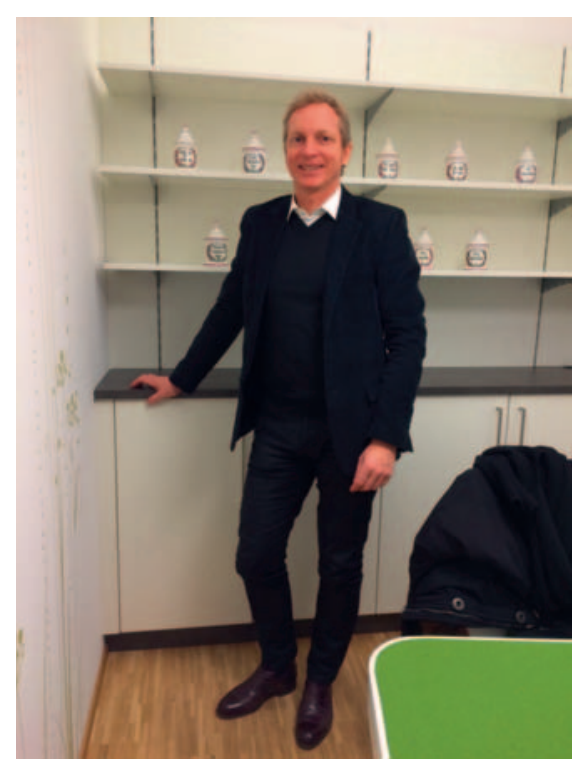

Abb. 4. Damien Verrier, Geschäftsführer von Schmidt-Nagel.

\section{Komplexhomöopathie}

Bei den umstrittenen KomplexHomöopathika, also Präparaten, die mehrere homöopathische Potenzen enthalten, geht Schmidt-Nagel einen pragmatischen Weg (siehe Kasten 1). Obwohl diese der ursprünglichen Lehre der Homöopathie widersprechen, hat sich Schmidt-Nagel dieser neuen Art von homöopathischen Präparaten nicht ganz verschlossen und bietet etwa 30 verschiedene homöopathische Mischungen an, die dem Anwender den Einstieg in die Klassische Homöopathie ermöglichen sollen. Darunter befinden sich Präparate mit ausschliesslich Tiefpotenzen wie z.B. Arnica complexe, das Arnica 9CH, Hypericum perforatum 9CH und Ruta graveolens $9 \mathrm{CH}$ enthält und das Feld von oberflächlichen Verletzungen abdeckt. Daneben findet man aber auch Komplexe, die aus höheren Potenzen zusammengesetzt sind, wie z.B. Euphrasia complexe mit Allium cepa 200K, Euphrasia officinalis $200 \mathrm{~K}$ und Sabadilla 200K, das gegen Allergien eingesetzt werden kann. 
Herr Verrier, vor Ihrer jetzigen Stelle als Geschäftsführer des Laboratoire D. Schmidt-Nagel SA waren Sie Kadermitglied grosser Firmen. Warum sind Sie auf das Niveau einer kleinen Homöopathiefirma hinabgesteigen?

Verrier: Einerseits war es nie meine Absicht, ganz gezielt in eine kleine Firma zu wechseln, aber die Qualität und die Substanz der Firma, die ich nun leite und dabei meinen Prinzipien treu bleiben kann, haben mich zu diesem Entschluss gebracht.

Andererseits sehe ich meine Qualitäten eher als Unternehmer denn als Kadermitglied eines Konzerns. Und vor meinem Engagement bei Schmidt-Nagel leitete ich während 8 Jahren einen internationalen, auf allen Kontinenten vertretenen Konzern, der mehrere Tausend Mitarbeitende beschäftigte und in einem völlig anderen Sektor tätig war. Jedoch fand ich dort aus verschiedenen Gründen weder das «Salz» noch die Motivation, die ich für mein Leben brauche. Nun, das Projekt, das mir von Frau Dominique Massotte vorgeschlagen wurde, entsprach perfekt meinen Vorstellungen, obwohl es in einem völlig anderen Geschäftsfeld liegt, das ich bisher überhaupt nicht kannte. Und für diesen Mut danke ich ihr hier nochmals! Schmidt-Nagel ist ein fantastisches Unternehmen mit einem sehr grossen Potenzial. Ich würde sagen, meine Aufgabe ist es, das Unternehmen auf der Basis seines Fundaments, das seine Stärke ausmacht, neu zu organisieren und zu entwickeln, um es so vorwärts zu bringen. Und schliesslich habe ich in der Homöopathie ein Gebiet entdeckt, das mich langsam, aber sicher begeistert.

\section{Was genau an der Homöopathie beginnt Sie langsam, aber sicher zu begeistern?}

Die Natur hat mir eine gute Gesundheit geschenkt, und ich musste mich bisher glücklicherweise praktisch nie behandeln lassen und Medikamente einnehmen. Und offen gestanden verstand ich von Homöopathie eigentlich nichts, aber auch nichts von Allopathie. Da ich aber viel Sport treibe und viel auf einen gesunden und ausgeglichenen Lebensstil achte, ergab diese Art von Medizin mit der Zeit für mich mehr Sinn. Ein weiterer wichtiger Grund ist die Tatsache, dass in meiner nächsten Umgebung eine Person ist, die seit Jahren mit Klassischer Homöopathie behandelt wird und bei der ich auf eindrückliche Weise die Wirksamkeit der Homöopathie feststellen konnte.

Nun, da ich in meinem beruflichen Alltag mit der Homöopathie verbunden bin, und weil ich eine sehr suchende Natur besitze, habe ich viel über Homöopathie gelesen, um diese Materie besser zu verstehen. Und ich habe jetzt auch die Möglichkeit, mich direkt mit der Wirksamkeit der Homöopathie auseinanderzusetzen. Daher kann ich bezeugen, dass die Homöopathie eine weltweit eingesetzte und überzeugende Therapierichtung ist. Die neuesten Fortschritte der Wissenschaft werden der Homöopathie endlich die ihr zukommende Legitimation geben. Das ist wirklich spannend.

Nur weil ich die Homöopathie fördere, bin ich aber noch lange kein Gegner der Allopathie. Wir sollten diese Debatten pro oder kontra Homöopathie aufgeben, weil sie keinen Sinn mehr ergeben. Wir sollten auf einem gemeinsamen Weg vorwärtsgehen, was dem Wohle des Patienten dient.

\section{Mit welchen grossen Herausforderungen wird Schmidt-Nagel gegenwärtig konfrontiert?}

Ich denke, es ist für die Homöopathie-Firmen sehr wichtig, neben ihren geschäftlichen Aktivitäten als Firmen aktiv an der Anerkennung und der Verbreitung der Homöopathie zu arbeiten. Dies geschieht neben der Kommunikation mit der breiten Öffentlichkeit durch die Weiterbildung von Gesundheitsfachleuten, durch unseren Beitrag zur Forschung und durch unsere Bemühungen, dass die Eigenheiten der Homöopathie von den Behörden des Gesundheitswesens akzeptiert werden. Denn man muss wissen, dass die gegenwärtigen regulatorischen Anforderungen diesen Eigenheiten in keiner Weise entgegenkommen oder sich diesen sogar anpassen. Ich denke hier vor allem an die Registrierung und Nachregistrierung von homöopathischen Substanzen, die beide sehr schwierig sind. 
Schmidt-Nagel engagiert sich bei diesen Problemen im Rahmen seiner Möglichkeiten. Das ist auch in unseren «Genen» angelegt, und man darf natürlich unsere ständigen Bemühungen nicht vergessen, unseren Kunden so viele homöopathische Substanzen wie möglich zur Verfügung zu stellen.

Was die Registrierungen betrifft, möchte ich betonen, dass ich für die Homöopathie eine ebenso klare und strenge Reglementierung verlange wie für die klassischen Medikamente. Wie könnte man sich denn einerseits zur Seriosität und Legitimität der Homöopathie bekennen, wenn man sich auf der anderen Seite von jeder Kontrolle und allen Normen befreien möchte?

Die Homöopathie ist eine in sich geschlossene Therapierichtung. Und sie muss einerseits für die Herstellung der homöopathischen Medikamente und andererseits für deren Anwendung ein Instrument haben, damit sie sich von dem entfernen kann, was ich Betrügerei nenne.

Noch eine Bemerkung zu den Aktivitäten der Homöopathie-Firmen - und letztlich zu allen Beteiligten der Homöopathie: Nachdem ich andere Firmen kennengelernt habe, meine ich, wir sollten uns vermehrt für einheitliche Anstrengungen einsetzen. Das soll auf nationaler, aber auch auf europäischer Ebene geschehen, um konzertant und mit einer einheitlichen Stimme aufzutreten. Dies wird uns zusätzlich stärken.

Der Vertrieb von homöopathischen Komplexmitteln ist lukrativer als jener von klassischen Homöopathika. Die Komplex-Homöopathie hat aber oft einen schlechten Ruf. Wo steht Schmidt-Nagel in diesem Spannungsfeld?

Schmidt-Nagel ist klar auf klassische Homöopathika spezialisiert. Das ergibt sich aus unserer Geschichte, unserer Kompetenz und unserem Geschäftsmodell.

Wir haben gewiss auch einige homöopathische Komplexe entwickelt und sind dabei sehr weise vorgegangen - denn wir wollten damit den Zugang zu unseren klassischen Homöopathika erleichtern. Unsere Bemühungen sind aber ganz klar auf die Einzelpotenzen, also auf die Klassische Homöopathie, ausgerichtet.

Dabei verfolgen wir aber die Tendenz und den Erfolg homöopathischer Komplexmittel, die in Selbstmedikation angewendet werden können, also von Endverbrauchern ohne Grundkenntnisse in Homöopathie. Auf dieses Modell stützen gewisse grosse Homöopathie-Firmen ihre Strategie und Entwicklung.

Jedoch stellt sich auch hier meine Beurteilung der manichäischen Logik entgegen, gemäss derer man ein Modell dem anderen entgegensetzt. Ich denke eher, beide sind nützlich und können sich ergänzen. Wenn ich die Anerkennung der Homöopathie fördern will, dann stört es mich nicht, auf dem Markt diese Art von Selbstmedikationshomöopathika zu sehen, solange die Anwender wissen - oder darüber aufgeklärt werden -, dass im Falle einer ernsthaften Erkrankung ein homöopathischer Arzt aufgesucht werden muss. Und dieser wird dem Kranken das für ihn geeignete homöopathische Einzelmittel verschreiben.

Zusammenfassend kann ich sagen, dass ich homöopathischen Komplexen für die Anwendung in der breiten Bevölkerung ziemlich wohlwollend gesinnt bin. Diese müssen dann aber im Bedarfsfall von homöopathischen Monodosen abgelöst werden, die von einem kompetenten Arzt verschrieben und von einem Apotheker erklärt werden.

Rein geschäftlich gedacht, ist es im gegenwärtigen Gesundheitswesen lukrativer und weniger risikobehaftet, sich mehr der Komplex-Homöopathie zuzuwenden. Wir von Schmidt-Nagel setzen aber auf die langfristigen Ziele.

\section{Was ist Ihre Botschaft an die Schweizer Ärzte und Apotheker?}

Unsere Hauptbotschaft lautet, dass sie auf Schmidt-Nagel zählen können und wir weiterhin Medikamente von hoher Qualität herstellen und den besten Service anbieten. Unsere Strategie und unsere Bemühungen sind darauf ausgerichtet. 